\title{
Wave chaos in the elastic disc
}

\author{
Niels Sondergaard *and Gregor Tanner ${ }^{\dagger}$ \\ School of Mathematical Sciences \\ University of Nottingham \\ University Park, Nottingham NG7 2RD, UK
}

November 2, 2018

\begin{abstract}
The relation between the elastic wave equation for plane, isotropic bodies and an underlying classical ray dynamics is investigated. We study in particular the eigenfrequencies of an elastic disc with free boundaries and their connection to periodic rays inside the circular domain. Even though the problem is separable, wave mixing between the shear and pressure component of the wave field at the boundary leads to an effective stochastic part in the ray dynamics. This introduces phenomena typically associated with classical chaos as for example an exponential increase in the number of periodic orbits. Classically, the problem can be decomposed into an integrable part and a simple binary Markov process. Similarly, the wave equation can in the high frequency limit be mapped onto a quantum graph. Implications of this result for the level statistics are discussed. Furthermore, a periodic trace formula is derived from the scattering matrix based on the inside-outside duality between eigen-modes and scattering solutions and periodic orbits are identified by Fourier transforming the spectral density. submitted to Physical Review E;
\end{abstract}

Version: 6th August 2002

PACS numbers: 03.65.Sq, 05.45.Mt, 46.40.-f, 46.40.Cd

\section{Introduction}

In the beginning of the 20th century, Debye studied the density of vibrational modes in a solid body in the context of his work on the heat capacity. He found that the average density is in leading order proportional to the volume of the body times the third power of the frequency. Corrections to Debye's result were found later involving contributions due to the surface of the body [1, 2]. The density of eigenfrequencies of a solid body contains apart form these smooth terms also oscillatory contributions, which build up the discrete spectrum of individual eigenmodes. These oscillatory corrections have been studied intensively over the last decade or so in the context of the Helmholtz and the Schrödinger equation. In the high frequency limit they are known to be related to periodic orbits of an underlying classical dynamics, that is, the ray dynamics in a billiard in the former or the Hamiltonian dynamics of the corresponding classical system in the latter

*e-mail: niels.sondergaard@nottingham.ac.uk

${ }^{\dagger}$ e-mail: gregor.tanner@nottingham.ac.uk 


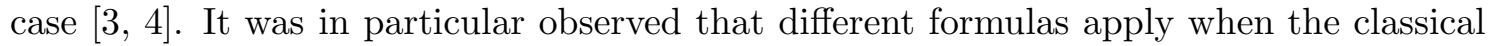
dynamics is integrable [5] or chaotic [6]. The relation between the wave equation and a related deterministic ray-dynamics is less obvious in elasticity. The wave equations are vectorial and different wave modes with differing wave velocities coexist. The notion of chaos or integrability needs to be reexamined here, which is the main purpose of this paper.

In what follows we shall assume that the elastic body consists of an isotropic material. To reduce the dimensionality of the problem, we will furthermore consider only bodies of the form of a thin plate or an infinite rod with constant cross section. The vibrations in the plate or rod decouple then into two classes, the in-plane and the anti-plane vibrations [7]; (for plates, this is only true as long as the wavelength is much smaller than the thickness of the plate). The problem is thus reduced to two spatial dimensions. We will focus here on the in-plane vibrations for which the wave equation is still vectorial which makes it more complex than say the scalar Helmholtz equation. The wave field can be decomposed into two polarizations, that is, pressure and shear waves, which have different wave speeds. The two polarizations couple at the boundary for physically relevant boundary conditions. An underlying ray dynamics emerging at high frequencies has similarly two types of rays traveling at different speeds and conversion between polarizations take place at the boundary. Ray conversion introduces a stochastic component into the dynamics and may lead to a large increase of possible ray trajectories compared to deterministic billiards for the same domain shapes [8].

We shall discuss mainly the case of a circular disc here, a separable problem due to the spherical symmetry. The case of elastic bodies without symmetries and fully chaotic classical ray dynamics has been discussed in [9] including a comparison with the semiclassical quantization of chaotic systems [3]. The scattering from two circular cavities in an elastic medium has been treated in [10]. The common idea is to write the spectral density as the trace of the Green's function which can in turn be expressed as sum over classical periodic orbits. We shall derive such a trace formula for the elastic disc and compare the results with the numerically calculated spectrum. We will furthermore show that the wave equation as well as the classical ray dynamics still posses a degree of 'randomness' due to the wave mixing at the boundary even though angular momentum is conserved.

The quantum spectra of systems whose classical dynamics is chaotic has been found to follow random matrix theory originally developed in nuclear physics, see for example [11, 12]. In the elastic case spectral statistics coinciding with RMT has been observed in experiments for rectangular and stadium shaped plates [13, 14, 15]. Recently, spectra of graphs have also be shown to behave quite similar to chaotic systems [16, 17]; they posses a trace formula for the spectral density and show random matrix statistics. We will make a connection between the ray dynamics in an elastic disc and the dynamics on a simple Markov graph, and will show how mode conversion effects the correlations in the eigenfrequency spectrum of the disc.

The paper is organized as follows; we shall first introduce the elastic wave equation and a high frequency approximation of its boundary element kernel in section 2. Next, the classical ray dynamics in a disc is discussed in section 3. In section 1 , the exact solutions of the wave equation for circular symmetries is derived and high frequency approximations are discussed. We will then study the so-called nearest neighbor spacing distribution for the disc spectrum in more detail. An expression for the oscillatory part of the level density in terms of periodic orbits will be derived from the scattering matrix in section 5 . 


\section{The elastic wave equation and short wavelength approxi- mations}

We shall consider the propagation of elastic deformations through an isotropic body. The partial differential equation in the frequency domain is the linear Navier-Cauchy equation [7, 18

$$
\mu \Delta(\mathbf{u})+(\lambda+\mu) \nabla(\nabla \cdot \mathbf{u})+\rho \omega^{2} \mathbf{u}=0,
$$

where $\mathbf{u}(\mathbf{x})$ is the displacement field in the body, $\lambda, \mu$ are the material dependent Lamé coefficients and $\rho$ is the density. We shall restrict ourselves to two-dimensional problems in what follows. A generalization of the results in this section to three dimensions is, however, straightforward. The two-dimensional wave equation describes in-plane deformations in plates or wave propagation in cylindrical bodies extending to infinity along one axis.

Introducing elastic potentials $\Phi$ and $\boldsymbol{\Psi}$ by using standard Helmholtz decomposition of the displacement field $\mathbf{u}$, that is,

$$
\mathbf{u}=\mathbf{u}_{p}+\mathbf{u}_{s} \quad \text { with } \quad \mathbf{u}_{p}=\nabla \Phi, \quad \mathbf{u}_{s}=\nabla \times \mathbf{\Psi},
$$

the Navier-Cauchy reduces to two Helmholtz equations for the potentials

$$
\left(\Delta+k_{p}^{2}\right) \Phi=0 ; \quad\left(\Delta+k_{s}^{2}\right) \Psi=0 .
$$

Here, $k_{p}$ and $k_{s}$ are the wave numbers for the pressure (or longitudinal) and shear (or transversal) wave component, respectively. The wave velocities relating the wave numbers to the frequency $\omega$ via the dispersion relation $k_{p, s}=\omega / c_{p, s}$ are different for the two different polarizations, on obtains

$$
c_{p}=\sqrt{\frac{\lambda+2 \mu}{\rho}} \quad c_{s}=\sqrt{\frac{\mu}{\rho}} .
$$

Note that the pressure wave speed is always larger than the shear wave velocity. It is this difference in wave speed which leads to the phenomenon of wave splitting in the ray dynamics on impact with a boundary, see fig. 1. The two wave equations (3) couple at the boundary, the details of the coupling depend on the boundary conditions. We shall in the following always consider free boundaries, that is, no forces act on the surface of the body. Forces acting on general surface elements are described in terms of the stress tensor

$$
\sigma_{i j}=\lambda \partial_{k} u_{k} \delta_{i j}+\mu\left(\partial_{i} u_{j}+\partial_{j} u_{i}\right)
$$

where the summation convention is used. Free boundary conditions correspond to

$$
\mathbf{t}(\mathbf{u})=\sigma(\mathbf{u}) \cdot \mathbf{n}=\mathbf{0}
$$

for the displacement field at the boundary where $\mathbf{n}$ denotes the normal to the boundary. The operator $\mathbf{t}$ refers to the traction. The traction operator (or traction matrix after representing it in a particular basis) for a circular boundary will be needed later to calculate the eigenfrequency spectrum of an elastic disc, and is explicitly derived in appendix $\mathrm{A}$.

Waves propagate freely inside the medium, that is, the pressure and shear component are decoupled and travel along straight lines. Wave splitting occurs at the boundary according to Snell's law

$$
\frac{c_{p}}{c_{s}}=\frac{\sin \theta_{p}}{\sin \theta_{s}},
$$




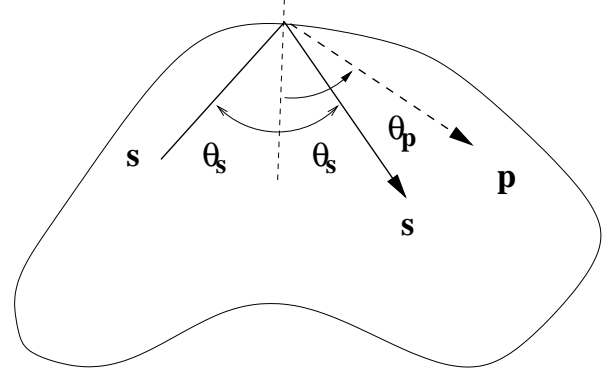

Figure 1: Wave splitting for in-plane waves at the boundary

where $\theta_{p}, \theta_{p}$ denote the angle of incident or reflection of the pressure and shear wave, respectively, measured with respect to the normal to the surface, see fig. 1. No mode conversion takes place for $s$-waves coming in at incident angles larger than a critical angle $\theta_{c}=\arcsin \left(c_{s} / c_{p}\right)$. The reflection coefficients at impact with a plane interface for free boundary conditions can be given in terms of an orthogonal $2 \times 2$ coefficient matrix $\boldsymbol{\alpha}$, [9]

$$
\begin{aligned}
\alpha_{p p} & =\frac{\sin 2 \theta_{s} \sin 2 \theta_{p}-\kappa^{2} \cos ^{2} 2 \theta_{s}}{\sin 2 \theta_{s} \sin 2 \theta_{p}+\kappa^{2} \cos ^{2} 2 \theta_{s}} \\
\alpha_{s s} & =\alpha_{p p} \\
\alpha_{p s} & =-\alpha_{s p} \quad \text { and } \quad \alpha_{p p}^{2}+\alpha_{p s}^{2}=1
\end{aligned}
$$

where $\alpha_{\pi \pi^{\prime}}$ relates an incoming wave of polarization $\pi \in\{s, p\}$ to an outgoing wave of polarization $\pi^{\prime}$ and $\kappa=c_{p} / c_{s}$. In the literature, often only the reflection coefficients for the displacement field $\mathbf{u}$ are given [7] related to the coefficient matrix $\alpha$ above by

$$
a_{\pi \pi^{\prime}}=\sqrt{\frac{c_{\pi} \cos \theta_{\pi}}{c_{\pi^{\prime}} \cos \theta_{\pi^{\prime}}}} \alpha_{\pi \pi^{\prime}} .
$$

Here, $\left|a_{\pi \pi^{\prime}}\right|^{2}$ is equivalent to the proportion of the energy density of the wave function undergoing transition from $\pi^{\prime} \rightarrow \pi$ whereas $\left|\alpha_{\pi \pi^{\prime}}\right|^{2}$ is the ratio of the corresponding energy fluxes normal to the boundary (with normal velocity $c_{\pi} \cos \theta_{\pi}$ ). The unitarity of $\alpha$ thus implies flux conservation normal to the boundary. The tangential energy flux is, however, not conserved for free boundary conditions due to the non-vanishing tangential stress $\sigma_{t t}$ giving rise to surface waves, (whereas $\sigma_{n n}=\sigma_{n t}=\sigma_{t n}=0$ at the boundary ) 19, 20.

We are interested here in solutions of the wave equations (11) in bounded domains in two dimensions. The set of eigenfrequencies is discrete and the solutions depend on the shape of the domain and on the boundary conditions. The wave equation is non-separable for typical domain shapes and numerical schemes, as for example boundary element methods (BEM), have to be employed to calculate the eigenfrequencies and corresponding wave-functions. The BEM is typically less straightforward in the elastodynamical case compared to applying it to the scalar Helmholtz equation. The integral kernels become hyper-singular for common boundary conditions as for example free boundaries and the displacement field is vectorial. Standard techniques to apply BEM to the Navier-Cauchy equations are described in [21].

Relatively simple expression for the boundary integral kernels can, however, be obtained when considering the high frequency limit. A generalization of Bogomolny's transfer operator method [22], derived originally for the Helmholtz equation in bounded domains, 
yields in two dimensions a boundary kernel in the form of a $2 \times 2$ matrix, that is,

$$
T\left(q, q^{\prime} ; \omega\right)=\sqrt{\frac{\omega}{2 \pi \mathrm{i}}} \sqrt{\left|\frac{\partial^{2} L}{\partial q \partial q^{\prime}}\right|}\left(\begin{array}{cc}
\alpha_{p p} & \alpha_{p s} \\
\alpha_{s p} & \alpha_{s s}
\end{array}\right)\left(\begin{array}{cc}
\sqrt{\frac{1}{c_{p}}} e^{i k_{p} L\left(q, q^{\prime}\right)-i \nu_{p} \frac{\pi}{2}} & 0 \\
0 & \sqrt{\frac{1}{c_{s}}} e^{i k_{s} L\left(q, q^{\prime}\right)-i \nu_{s} \frac{\pi}{2}}
\end{array}\right)
$$

where $q, q^{\prime}$ denote points on the boundary of the domain. $L\left(q, q^{\prime}\right)$ is the distance between $q$ and $q^{\prime}$ in the two-dimensional $(x, y)$ - plane and the reflection coefficients are defined in (6). The additional phases $\nu_{p, s}$ are Maslov indices which count the number of caustics along the path for each polarization [3]. Approximations to the eigenfrequencies are then obtained by solving

$$
\operatorname{det}(\mathbf{1}-\mathbf{T}(\omega)) \stackrel{!}{=} 0 .
$$

The transfer operator (8) can be viewed as a discrete wave propagator acting on boundary wave functions by mapping outgoing two-component wave vectors at a point $q$ on the boundary into outgoing wave vectors at $q^{\prime}$. Wave mixing at the boundary enters through the matrix $\boldsymbol{\alpha}(q)$. Snell's law (5) is obtained by considering the two-step operator

$$
T^{2}\left(q, q^{\prime \prime} ; \omega\right)=\oint d q^{\prime} T\left(q, q^{\prime} ; \omega\right) T\left(q^{\prime}, q^{\prime \prime} ; \omega\right)
$$

in stationary phase approximation. Considering $n$ step operators $T^{n}$, one can derive periodic orbit trace formulas as presented in [9], see also section 5.

The transfer operator is in many respect a fairly crude approximation of the true BEM. It is the leading order term in a $1 / \omega$ expansions of the exact boundary integral kernel and does in particular not contain evanescent contributions. It can therefore not reproduce boundary effects like surface waves as well as diffraction or higher order mode mixing corrections. It is, however, a natural starting point to investigate the connection between the wave dynamics in elastic media of finite size and an underlying ray dynamics which includes ray splitting.

In the following, we shall study the billiard with probably the most simple geometry, namely an elastic disc. Even though the wave equation is separable for this particular shape, there is some degree of wave chaos in this system, which can be traced back to chaotic components of an underlying ray dynamics.

\section{Classical ray dynamics for circular domains}

In this section, we shall discuss in more detail the ray dynamics in isotropic media of general shape and in particular for two-dimensional circular domains.

We will adopt the following convention for a ray-trajectory in an elastic isotropic medium: a trajectory is at any instant in time given by its position and momentum as well as its polarization being either of $p$ or $s$ type. We may identify the wave numbers $k_{\pi}=\omega / c_{\pi}, \pi \in\{p, s\}$ as the momenta of polarization $\pi$ with $k_{s}=\kappa k_{p}$. A ray travels along straight lines between impacts with the boundary. At the boundary, it stays in a given polarization or undergoes mode conversion with probability

$$
t_{p p}=t_{s s}=\left|\alpha_{p p}\right|^{2}, t_{p s}=t_{s p}=\left|\alpha_{p s}\right|^{2},
$$

where the reflection coefficients are given in (6). The angle of reflection depends on whether mode conversion takes place or not via Snell's law and so does the momentum. 
A trajectory is uniquely determined after fixing its initial position and momentum and an infinite sequence of polarizations $\pi_{1}, \pi_{2}, \ldots, \pi_{i} \ldots \in\{s, p\}$ reflecting the probabilistic nature of the dynamics. The dynamics in an elastic isotropic medium is thus taking place on two different energy sheets with energies $E_{p}=k_{p}^{2}$ and $E_{s}=k_{s}^{2}$ with $E_{p}<E_{s}$. The dynamics on each sheet is deterministic, jumps from one sheet to the other may occur at the boundaries.

We shall now turn to the dynamics in a circular disc with radius $a$. The angular momentum $L$ is conserved at impact with a boundary both for rays staying in a given polarization and those undergoing mode conversion which follows directly from (5), that is,

$$
L=z_{p} \sin \theta_{p}=z_{s} \sin \theta_{s}=\text { const },
$$

where we set $z_{p}=a k_{p}$ and $z_{s}=a k_{s}$. The maximal angular momentum possible for fixed frequency $\omega$ is $\left|L_{\max }\right|=z_{s}$, no wave splitting occurs for $z_{p} \leq|L| \leq z_{s}$. The dynamics in a disc follows a simple scaling relation and can be characterized in terms of the dimensionless impact parameters $b_{\pi}=L / z_{\pi}$ with $b_{p}=\kappa b_{s}$. The mode splitting regime is characterized by $\left|b_{p}\right|<1$, pure $s$ - rays exist for $1<\left|b_{p}\right|<\kappa$. A trajectory takes on at most two different angles of reflection $\theta_{p}$ and $\theta_{s}$ with $\sin \theta_{\pi}=b_{\pi}$.

In the language of dynamical systems theory, one may say that the dynamics on each energy sheet is integrable, that is, trajectories for fixed $L$ and a given polarization are confined to a two-dimensional manifold in phase space with the topology of a torus. Mode conversion couples two specific tori characterized by $\left(E_{p}, L\right)$ and $\left(E_{s}, L\right)$ and transitions are possible between these two tori only, see fig. 2 a. The total dynamics is thus not ergodic.

For $\left|b_{p}\right|<1$, the same initial condition in phase space does, however, lead to an exponentially increasing number of possible solutions due to the transitions between the energy sheets. This leads, for example, to an exponential increase in the number of periodic ray trajectories with increasing length, a phenomenon usually associated with classical chaos. The latter follows directly from the periodic orbit condition

$$
n_{p} \Delta \varphi_{p}+n_{s} \Delta \varphi_{s}=2 \pi m, \quad n_{p}+n_{s}=n \geq 2 m .
$$

Here, $\Delta \varphi_{\pi}$ is the change in the azimuthal angle for a ray with polarization $\pi$ between two reflections. The azimuthal angle and the angle of incidence are related through the relation $\Delta \varphi_{\pi}=\pi-2 \theta_{\pi}$. The integer indices $n_{\pi}$ correspond to the number of ray segments with polarization $\pi$ and $m$ is the number of rotations around the center. The number $N(n)$ of periodic orbits with $n$ reflections (including permutations of the polarizations) increases thus exponentially like

$$
N(n) \sim \frac{n}{2} 2^{n}
$$

The fact, that these orbits (apart from the $m=0$ case) all form continuous families is, however, a feature known from integrable dynamics.

The dynamics becomes particular simple when we restrict our considerations to the motion in the radial coordinate only. The dynamics in $r$ is one-dimensional in each sheet and bound away from the center due to the centrifugal potential $L^{2} / 2 r^{2}$, see fig. 2 a. Transitions between the sheets occur at the boundary $r=a$ for $\left|b_{p}\right|<1$. The boundary map for the radial dynamics for fixed $b_{p}$ is thus a simple stochastic process which may be described in terms of a graph with two loops of the form shown in fig. $2 \mathrm{~b}$. The transition rates (10) which again depend only on the parameters $b_{p}$ and $\kappa$ define a Markov process 
a)

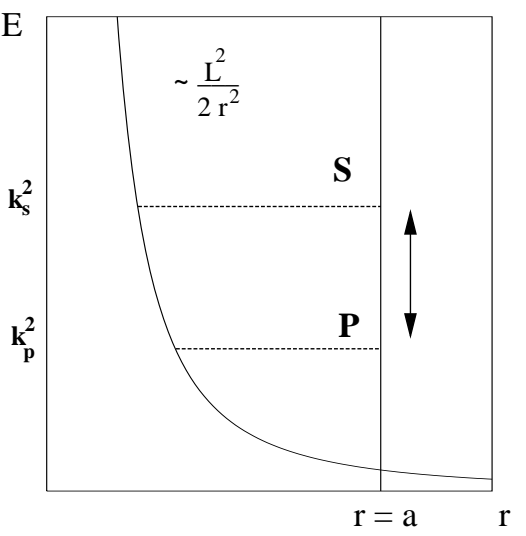

b)

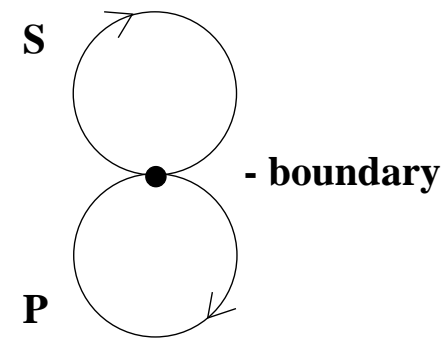

Figure 2: a) Radial dynamics in the disc takes place on two energy sheets $E_{p}=k_{p}^{2}$ and $E_{s}=k_{s}^{2}=\kappa^{2} E_{p}$; transitions take place at the boundary $r=a$.

b) The boundary map is equivalent to the probabilistic dynamics of a Markov process on a binary graph.

on this graph with topological entropy $h_{t}=\log 2$ and exponential decay of correlation for $t_{p p} \neq 0$ or 1 . The chaotic component of the dynamics in the elastic disc is thus a two-level stochastic process.

\section{The elastic disc - exact results and the high-frequency limit}

The wave equation for a disc of radius $a$ is separable independent of the boundary conditions. We will briefly discuss the exact solutions in the case of free boundaries and make a connection between the eigenfrequencies of the interior problem and the spectrum of the scattering matrix for the corresponding exterior problem. Details are referred to the appendices A and B. It will in particular be shown that the scattering matrix is equivalent to the transfer operator (8) in the high-frequency limit.

\subsection{The scattering matrix}

The elastic wave equation can for circular domains in two dimensions be solved in terms of the basis functions

$$
\psi_{l}(r, \varphi)=J_{l}\left(k_{\pi} r\right) e^{i l \varphi},
$$

where $J_{l}\left(k_{\pi} r\right)$ is the $l$ th order Bessel function with $\pi=p$ or $s$ and $\varphi$ is the azimuthal angle. The separability of the wave equation reflects the conservation of angular momentum in the classical ray dynamics; we obtain as usual that the angular momentum takes on only integer values $l$.

Applying free boundary conditions to a displacement wave vector obtained from the potentials (13) for fixed $l$ leads to the secular equation, see appendix A,

$$
\operatorname{det}\left(\mathbf{t}_{l}\right)(\omega)=0
$$


where the traction matrix $\mathbf{t}$ is given as

$$
\mathbf{t}_{l}=\left(\begin{array}{cc}
\left(l^{2}-\frac{1}{2} z_{s}^{2}\right) J_{l}\left(z_{p}\right)-z_{p} J_{l}^{\prime}\left(z_{p}\right) & i l\left(J_{l}\left(z_{p}\right)-z_{p} J_{l}^{\prime}\left(z_{p}\right)\right) \\
i l\left(J_{l}\left(z_{s}\right)-z_{s} J_{l}^{\prime}\left(z_{s}\right)\right) & z_{s} J_{l}^{\prime}\left(z_{s}\right)-\left(l^{2}-\frac{1}{2} z_{s}^{2}\right) J_{l}\left(z_{s}\right)
\end{array}\right)
$$

and $z_{\pi}=k_{\pi} a$. The condition (14) can be rewritten in terms of the scattering matrix for the outside problem, that is, for in-plane wave - scattering in an infinite plate with a circular hole. This connection is known as the inside-outside duality [23] between eigensolutions of the interior problem and transparent scattering solutions. The scattering matrix for fixed angular momentum $l$ is given as [19]

$$
\mathbf{S}_{l}=-\mathbf{t}_{l}^{-} \cdot\left(\mathbf{t}_{l}^{+}\right)^{-1}
$$

see also appendix $\mathrm{B}$, where $\mathbf{t}_{l}^{+}, \mathbf{t}_{l}^{-}$are obtained from the traction matrix (15) by replacing the Bessel function and its derivatives by incoming and outgoing Hankel functions. Using $J_{l}(z)=\left(H_{l}^{(+)}(z)+H_{l}^{(-)}(z)\right) / 2$ which implies the same identity for the corresponding traction matrices, that is,

$$
\mathbf{t}_{l}=\frac{1}{2}\left(\mathbf{t}_{l}^{+}+\mathbf{t}_{l}^{-}\right)
$$

the eigenfrequency condition (14) can be written as

$$
\begin{aligned}
0=\operatorname{det}\left(\mathbf{t}_{l}\right) & =\frac{1}{4} \operatorname{det}\left(\mathbf{t}_{l}^{+}\right) \operatorname{det}\left(\mathbf{1}+\mathbf{t}_{l}^{-} \cdot\left(\mathbf{t}_{l}^{+}\right)^{-1}\right) \\
& \equiv \frac{1}{4} \operatorname{det}\left(\mathbf{t}_{l}^{+}\right) \operatorname{det}\left(\mathbf{1}-\mathbf{S}_{l}\right) .
\end{aligned}
$$

The zeros of the first factor in (18) are related to the resonances for exterior scattering at a circular cavity and are all in the lower complex $z$ - plane. An eigenfrequency for the interior problem of the disc implies that the scattering matrix at the same frequency has an eigenvalue 1. That is, a scattering solution exists for which the obstacle, here the disc, is transparent. This principle holds for general shapes [23].

\subsection{The scattering matrix in the high frequency limit}

In the following we shall derive an approximation to the scattering matrix in the high frequency limit.

The mode conversion regime $\left|b_{p}\right|<1$ : In the energy - angular momentum regime for which the impact parameter $\left|b_{p}\right|=|L| / z_{p}<1$, the Hankel functions entering $\mathbf{t}^{ \pm}$may be split in terms of phases and amplitudes using the oscillatory Debye approximation; one obtains to leading order (appendix B),

$$
\mathbf{S}_{l} \approx\left(\begin{array}{cc}
e^{-i \phi_{p}} & 0 \\
0 & e^{-i \phi_{s}}
\end{array}\right) \cdot \boldsymbol{\alpha}_{l} \cdot\left(\begin{array}{cc}
e^{-i \phi_{p}} & 0 \\
0 & e^{-i \phi_{s}}
\end{array}\right)
$$

with

$$
\phi_{\pi}=z_{\pi}\left(\sqrt{1-b_{\pi}^{2}}-b_{\pi} \arccos \left(b_{\pi}\right)\right)-\frac{\pi}{4}
$$

and $\boldsymbol{\alpha}_{l}$ is the unitary matrix of reflection coefficients defined in (6) with angles of incidence fixed by the angular momentum condition (11) with $L=l$. We thus obtain for transitions between polarizations $\pi, \pi^{\prime}$

$$
\mathbf{S}_{l}\left(\pi \rightarrow \pi^{\prime}\right) \approx \alpha_{\pi \pi^{\prime}} \cdot e^{-i\left(\phi_{\pi}+\phi_{\pi^{\prime}}\right)}
$$


Note that the unitarity of the $S$ - matrix is preserved in this approximation. This is not true in general for short wavelength approximations and results here from the quasi one-dimensionality of the dynamics.

Regime of no mode conversion $1<\left|b_{p}\right|<\kappa$ : For angular momenta corresponding to incident angles larger than the critical angle or $\left|b_{p}\right|>1$, a somewhat different treatment needs to be employed. Here the exponential Debye expansion must be used for the pressure wave leading to an $S$ - matrix of the form

$$
\mathbf{S}_{l} \approx\left(\begin{array}{cc}
1 & 0 \\
0 & \alpha_{s s} e^{-2 i \phi_{s}}
\end{array}\right)
$$

with a reflection coefficient (in agreement with the plane interface result)

$$
\alpha_{s s}=-\frac{Z_{l}^{*}}{Z_{l}}
$$

and

$$
Z_{l}=1+\cos 4 \theta_{s}+i 8 \cos \theta_{s} \sin ^{2} \theta_{s} \sqrt{\sin ^{2} \theta_{s}-1 / \kappa^{2}} .
$$

Here, the boundary conditions lead to a pure phase shift dependent on the angle of incidence. There is no attenuation associated with this reflection contrary to the wave splitting case. The phase shift is due to a coupling to a surface longitudinal wave 18.

The transfer matrix for $\left|b_{p}\right|<1$ : By parametrising the boundary in terms of the azimuthal angle $\varphi$, one obtains for the transfer operator (8) in a circular domain

$$
T\left(\varphi, \varphi^{\prime} ; \omega\right)=\sqrt{\frac{\omega}{2 \pi i}} \sqrt{\frac{a}{2} \sin \frac{\Delta \varphi}{2}} \boldsymbol{\alpha}(\Delta \varphi)\left(\begin{array}{cc}
\sqrt{\frac{1}{c_{p}}} e^{i k_{p} d} & 0 \\
0 & \sqrt{\frac{1}{c_{s}}} e^{i k_{s} d}
\end{array}\right),
$$

with $\Delta \varphi=\left|\varphi-\varphi^{\prime}\right|$ and $d$ is the distance between two points $\varphi, \varphi^{\prime}$ on the boundary, that is,

$$
d\left(\varphi, \varphi^{\prime}\right)=2 a \sin \frac{\Delta \varphi}{2} .
$$

The transfer operator depends only on the difference $\Delta \varphi$ and block-diagonalises with respect to the Fourier basis $\mid l>=\exp (i l \varphi) / \sqrt{2 \pi}$ for integer $l$. One obtains after evaluating the second integral by stationary phase

$$
<l|\mathbf{T}| l^{\prime}>=\delta_{l l^{\prime}} \boldsymbol{\alpha}_{l}\left(\begin{array}{cc}
e^{2 i \phi_{p}} & 0 \\
0 & e^{2 i \phi_{s}}
\end{array}\right),
$$

where the phases

$$
\phi_{\pi}=\frac{1}{2} k_{\pi} d\left(\Delta \varphi_{\pi}\right)-l \frac{\Delta \varphi_{\pi}}{2}-\frac{1}{4} \pi
$$

are taken at the stationary phase point

$$
a k_{\pi} \cos \frac{\Delta \varphi_{\pi}}{2}=a k_{\pi} \sin \theta_{\pi}=l
$$

which is the angular momentum condition (11). The phases $\phi_{\pi}$ in (27) coincide with (20) after inserting the stationary phase condition $\Delta \varphi_{\pi} / 2=\arccos b_{\pi}$, Eq. (28). The S-matrix in the approximation (19) is thus equivalent to the hermitian conjugate of the T-matrix up to a simple transformation in terms of a unitary diagonal matrix. In particular the eigenfrequency conditions $\operatorname{det}(\mathbf{1}-\mathbf{T}) \approx \operatorname{det}(\mathbf{1}-\mathbf{S})=0$ coincide in the high frequency limit. 

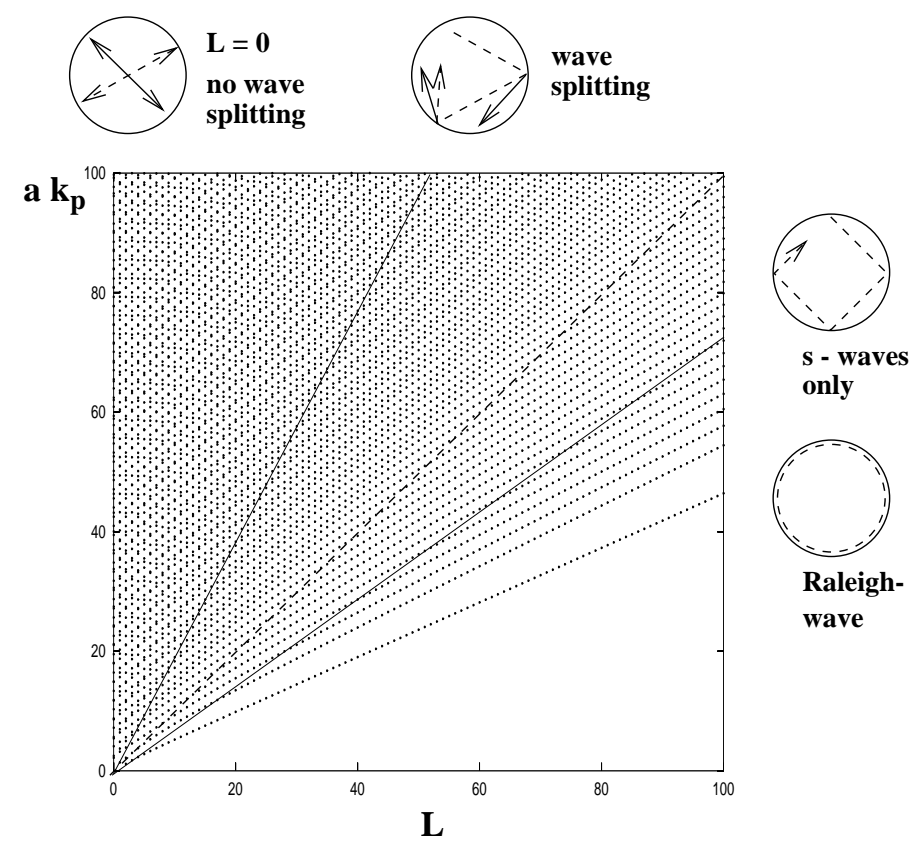

Figure 3: The eigenspectrum of the disc for aluminum with $\kappa=2.05$; the wavenumber $z_{p}=a k_{p}$ of an eigenfrequency is plotted versus the angular momentum $l$. Eigenfrequencies with $a k_{p}<l$ (or $\left|b_{p}\right|>1$ ) are pure shear states, mode mixing occurs for $a k_{p}>l$. The lowest states in each $l$ - series are due to surface waves (Rayleigh - waves).

The mean density of eigenfrequencies for fixed $l$ : The mean density of eigenfrequencies $\bar{d}_{l}$ for fixed $l$ can be obtained from the scattering matrix $\mathbf{S}_{l}$ [23], that is,

$$
\bar{d}_{l}\left(k_{p}\right)=\frac{1}{2 \pi i} \frac{d}{d k_{p}} \log \operatorname{det}\left(\mathbf{S}_{l}^{\dagger}\right) .
$$

Inserting the high frequency limit of the $S$ matrix (19) for $\left|b_{p}\right|<1$ one obtains to leading order

$$
\bar{d}_{l}\left(k_{p}\right)=\frac{1}{\pi} \frac{d}{d k_{p}}\left(\phi_{p}+\phi_{s}\right)=\frac{a}{\pi}\left(\sqrt{1-b_{p}^{2}}+\kappa \sqrt{1-b_{s}^{2}}\right) .
$$

Note that the mean level density depends on $k_{\pi}$ and $l$ only via the impact parameters $b_{\pi}$. Equivalently, we obtain from (22) for $1<\left|b_{p}\right|<\kappa$

$$
\bar{d}_{l}\left(k_{p}\right)=\frac{1}{\pi} \frac{d}{d k_{p}} \phi_{s}=\frac{a}{\pi} \kappa \sqrt{1-b_{s}^{2}} .
$$

\subsection{Statistical properties of the eigenspectrum}

We saw in the last section that a transition takes place at $\left|b_{p}\right|=|L| / k_{p} a=1$ between a pure shear wave regime with $\left|b_{p}\right|>1$ and a mode mixing regime with $\left|b_{p}\right|<1$. This transition is reflected in the ray dynamics, see section 3. Whereas only one family of (shear-) trajectories exists for fixed $b_{p}$ with $\left|b_{p}\right|>1$, there are infinitely many such families for $\left|b_{p}\right|<1$ and their number increases exponentially with the length of the trajectories. Such a phenomenon is reminiscent to the behavior typically found for chaotic classical 
dynamics. It was observed in section 3 that the dynamics in the mode mixing regime can indeed be described by a stochastic Markov process on a two-loop graph, see fig. $2 \mathrm{~b}$. In the limit $b_{p} \rightarrow 0$, however, the transition rates $t_{p p}=t_{s s}$ approach one and the two modes decouple again leaving only two possible ray-trajectories.

All these regimes should manifests itself also in the spectrum of the elastic disc. Spectral correlations are known to be particularly sensitive to the degree of chaos present in an underlying classical (ray-) dynamics [11, 12]. A popular statistical measure is the socalled nearest neighbour spacing distribution $P(s)$ giving the probability of finding two adjacent eigenvalues of the spectrum (unfolded to mean level separation one) a distance $s$ apart. $P(s)$ follows a Poisson distribution for completely uncorrelated spectra, but has been conjectured to coincide with the results obtained for ensembles of random Hermitian matrices for completely chaotic dynamics.

In fig. 3, the spectrum obtained from the exact eigenfrequency condition (15) for an aluminum disc with $\kappa=2.05$ is shown. Here, the wavenumber $z_{p}=a k_{p}$ of an eigenvalue with angular momentum $l$ is plotted. One notices a difference in the mean density of eigenvalues for fixed $l$ above and below the diagonal $\left|b_{p}\right|=|l| / z_{p}=1$, see Eqs. (29) and (30). The lowest eigenfrequency in each $l$ - series can be attributed to a surface (or Rayleigh-) wave. Mode mixing occurs for $\left|b_{p}\right|<1$ or $a k_{p}>L$.

First we look at $P(s)$ for the total spectrum which is obtained by projecting the different $l$ - eigenfrequency series in fig. 30 onto the $z_{p}$-axis. One finds indeed good agreement with Poisson statistics as shown in fig. 4 a. This reflects the fact that the ray-dynamics in the elastic disc is not ergodic but restricted to manifolds with fixed angular momentum $L$ which have the form of a single torus for $\left|b_{p}\right|>1$ or two coupled tori for $\left|b_{p}\right|<1$. The eigenfrequencies for different $l$ - series are thus uncorrelated, which leads to vanishing correlations in the full spectrum for large $z_{p}$ after projection onto the $z_{p}$-axis.

In order to see the influence of wave mixing on the spectrum, we need to study spectral correlations within a given $l$ series. Indeed, the wave - dynamics for fixed $l$ is given by the $2 \times 2$ transfer matrix Eq. (26) (or the equivalent matrix for $\left|b_{p}\right|>1$ obtained from (22)) being of the form

$$
\mathbf{T}_{l}\left(z_{p}\right)=\boldsymbol{\alpha}_{l}\left(b_{p}\right) \cdot\left(\begin{array}{cc}
e^{i z_{p}} \tilde{\phi}_{p}\left(b_{p}\right) & 0 \\
0 & e^{i z_{p} \tilde{\phi}_{s}\left(b_{p}\right)}
\end{array}\right)
$$

The transfer matrix $\mathbf{T}_{l}$ consisting of a unitary transition matrix $\boldsymbol{\alpha}$ times a diagonal matrix is typical for propagation on quantum graphs as studied in 16, 17]. The phases in the diagonal matrix $\tilde{\phi}_{p}, \tilde{\phi}_{p}$ are thereby interpreted as the lengths of the bonds in the graph, here the two loops in fig. $2 \mathrm{~b}$. As we change $z_{p}$, we expect the correlations within a given $l$ series to change according to the degree of wave-mixing possible, that is, to the degree, that $t_{p p}$ deviates from zero or one. Note that the transition amplitude $t_{p s}$ allowing for transitions between $p$ and $s$ waves goes to zero both in the limit $b_{p} \rightarrow 0$ and $b_{p} \rightarrow 1$, see Eq. (6); the wave modes decouple in these limits. We expect furthermore, that correlations are locally the same for different $l$ series with $b_{p}$ fixed, that is, along straight lines in fig. 1 with $b_{p}=$ const. We therefore study the nearest neighbour spacing distribution $P(s)$ for eigenfrequencies lying in a window given by the intersection of the cone $b_{p} \pm \Delta b_{p}$ and the line $L=l=$ const; the observed distributions $P\left(s, b_{p}\right)$ are indeed independent of $l$ and we may average $P\left(s, b_{p}\right)$ over different $l$ - series.

The result is shown in fig. $1 \mathrm{~b}$ for three different values of $b_{p}$ and $\Delta b_{p}=0.01$. In addition, the value of $t_{p p}$ as a function of $b_{p}$ is given; maximal mixing corresponds to 
a)

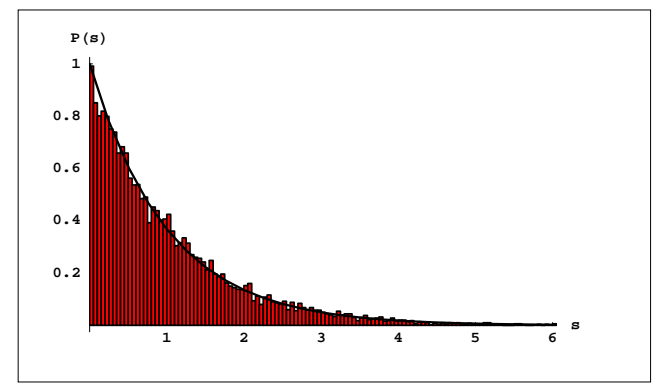

b)

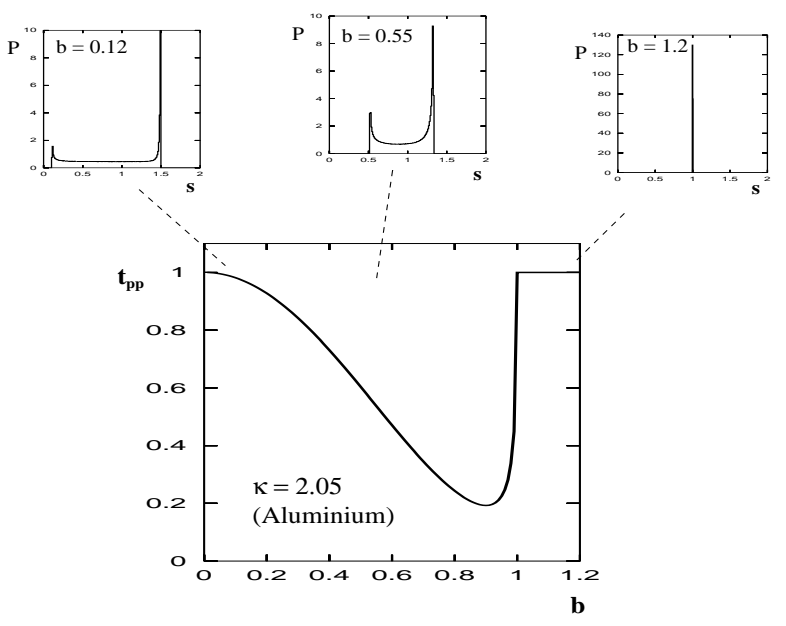

Figure 4: Statistical properties of the eigenspectrum for aluminum with $\kappa=2.05$. a) the nearest neighbour spacing distribution (NNS) for all levels; b) NNS distributions for fixed impact parameter $b_{p}$ together with the transition probabilities $t_{p p}$ as a function of $b_{p}$. The NNS is obtained for stretches of eigenvalues lying in a range $b_{p} \pm \Delta b_{p}$ for angular momenta from $l=300-3000$;

$t_{p p}=0.5$. The distributions are neither similar to the Poisson distribution, nor to any of the random matrix distributions. This non-universal behavior is typical for graphs with only a few bonds 24]. One notices, however, that a gap is opening up in $P(s)$ for small $s$ as $b_{p}$ increases from zero. This can be interpreted as level-repulsion due to mode-mixing which increases as $t_{p p}$ deviates from one. For $b_{p} \rightarrow 1$ a different effect sets in; the pressure mode is suppressed and we witness the transition form a two mode to a one mode wave dynamics with equidistant eigenfrequencies.

\section{$5 \quad$ Eigenfrequency density and a periodic orbit trace formula}

So far we have shown that the traction matrix as well as the scattering and transfer matrix can be brought into block-diagonal form where each $2 \times 2$ block produces the spectrum for 
fixed angular momentum $l$. In this section, we will make an explicit connection between the full spectrum and periodic trajectories in the elastic disc by looking at the total spectral density

$$
d(\omega)=\sum_{i} \delta\left(\omega-\omega_{i}\right)
$$

where the sum runs over all eigenfrequencies (in terms of the angular velocity $\omega$ ) of the disc.

The spectral density can quite generally be written in terms of a smooth part and oscillatory contributions, the latter containing the period orbit contributions, that is,

$$
d(\omega)=d_{\text {smooth }}(\omega)+d_{o s c}(\omega)
$$

The smooth part gives the mean density of states which may be obtained using

$$
d_{\text {smooth }}(\omega)=\frac{d N_{\text {smooth }}}{d \omega}
$$

where $N_{\text {smooth }}$ is the mean part of the spectral counting function $N(\omega)$ giving the number of levels below $\omega$. General results for the smooth part of the counting function of isotropic elastic media with free boundary conditions can be given [1, 2], that is,

$$
N_{\text {smooth }}(\omega) \approx \frac{c_{p}^{-2}+c_{s}^{-2}}{4 \pi} S \omega^{2}+\frac{\beta}{4 \pi c_{s}} L \omega+\mathrm{o}(\omega)
$$

with

$$
\beta=4 \eta-3+\kappa+4 I / \pi
$$

and

$$
I=\int_{1 / \kappa}^{1} \arctan \frac{\left(2-1 / \xi^{2}\right)^{2}}{4 \sqrt{\left(1-\frac{1}{\kappa^{2} \xi^{2}}\right)\left(\frac{1}{\xi^{2}}-1\right)}} d \xi .
$$

Here $S, L$ are the surface area and the perimeter of the domain and finally $\eta=c_{R} / c_{s}$ with $c_{R}$ the Rayleigh wave velocity [7]. The leading term corresponds to the available phase space volume whereas the next order term contains corrections due to surface states. In contrast to the scalar Helmholtz equation for which the expansion of $N_{\text {smooth }}$ can be worked out to arbitrary order in principle [25] only the first two terms are known in elastodynamics at present.

The fluctuating part of the density of states which contains all the information about individual eigenfrequencies of the interior problem can be written in terms of the scattering matrix of the outside problem [23, 26]. One obtains

$$
d_{o s c}(\omega)=\frac{1}{\pi} \operatorname{Im} \sum_{n=1}^{\infty} \frac{1}{n} \frac{d}{d \omega} \operatorname{Tr}\left(\mathbf{S}^{n}(\omega)\right)^{\dagger} .
$$

In the high frequency limit this term is related to periodic ray trajectories in the disc as will be shown below. 


\subsection{Oscillatory part of the density of eigenfrequencies}

We will first derive a periodic orbit expression for $\operatorname{Tr} \mathbf{S}^{n}$ in the high frequency limit $\omega \gg 1$ using the block-diagonal form of the scattering matrix and the approximation (19), that is,

$$
\begin{aligned}
\operatorname{Tr} \mathbf{S}^{n}(\omega) & =\sum_{|l| \leq l_{\max }} \operatorname{Tr} \mathbf{S}_{l}^{n} \\
& \approx \sum_{|l| \leq l_{\max }} \sum_{\boldsymbol{\pi}_{n}} \mathcal{A}_{\boldsymbol{\pi}_{n}} e^{-i 2\left(n_{p} \phi_{p}+n_{s} \phi_{s}\right)} .
\end{aligned}
$$

Here, $l_{\max } \sim z_{s}$ denotes the maximal angular momentum and the second sum runs over all binary symbol strings $\pi_{n}=\pi_{1} \pi_{2} \ldots \pi_{n}$ of length $n$ with $\pi_{i} \in\{p, s\}$. The amplitude $\mathcal{A}_{\boldsymbol{\pi}_{n}}$ is obtained as product over reflection coefficients (6), that is

$$
\mathcal{A}_{\boldsymbol{\pi}_{n}}=\prod_{i=1}^{n} \alpha_{\pi_{i} \pi_{i+1}}
$$

$\phi_{\pi}$ are the phases defined in (20) and $n_{p}, n_{s}$ equal the number of times the symbol $p, s$ appears in $\boldsymbol{\pi}_{n}$. The sum over binary symbol strings for fixed $l$ is equivalent to a sum over all periodic paths in the binary graph fig. 2. Note that the reflection coefficients as well as the phases $\Phi_{\pi}$ depend explicitly on $l$ and $\omega$.

Next, we use Poisson summation to write the sum over $l$ as

$$
\begin{aligned}
\operatorname{Tr} \mathbf{S}^{n} & \approx \sum_{\boldsymbol{\pi}_{n}} \sum_{|l| \leq l_{\max }} \mathcal{A}_{\boldsymbol{\pi}_{n}} e^{-2 i\left(n_{p} \phi_{p}+n_{s} \phi_{s}\right)} \\
& =\sum_{\boldsymbol{\pi}_{n}} \sum_{m=-\infty}^{\infty} \int_{-l_{\max }}^{l_{\max }} d l \mathcal{A}_{\boldsymbol{\pi}_{n}} e^{-2 i\left(n_{p} \phi_{p}+n_{s} \phi_{s}\right)-2 \pi i m l}
\end{aligned}
$$

Evaluating the integrals by stationary phase using

$$
\frac{d \phi_{\pi}}{d l}=-\arccos \left(l / z_{\pi}\right)=-\frac{\Delta \varphi_{\pi}}{2}
$$

where $\Delta \varphi_{\pi}$ is the angle spanned by a ray-segment with polarization $\pi$ between two reflections, we obtain the stationary phase condition

$$
\Delta \varphi_{\text {total }}=n_{p} \Delta \varphi_{p}+n_{s} \Delta \varphi_{s}=2 \pi m .
$$

This is precisely the periodic orbit condition (12), that is, only those angular momenta $l^{*}=z_{\pi} \cos \frac{1}{2} \Delta \varphi$ contribute significantly, for which a periodic orbit exists at frequency $\omega$. The second derivative of the phases in (35) is

$$
-\frac{d\left(\Delta \varphi_{\text {total }}-2 \pi m\right)}{d l}=2\left(\frac{n_{p}}{z_{p} \cos \theta_{p}}+\frac{n_{s}}{z_{s} \cos \theta_{s}}\right)
$$

with $\theta_{\pi}$, the angle of incident and $\cos \theta_{\pi}=\sin \frac{1}{2} \Delta \varphi_{\pi}$.

After evaluating the phases $\Phi_{\pi}$ in (35) at the stationary phase point $l^{*}$, one obtains for the total phase

$$
2\left(n_{p} \Phi_{p}+n_{s} \Phi_{s}\right)+2 \pi l^{*} m=n_{p} k_{p} d_{p}+n_{s} k_{s} d_{s}-n \frac{\pi}{2}=\omega T-n \frac{\pi}{2}
$$


with $n=n_{p}+n_{s}$ and $d_{\pi}=2 a \sin \frac{1}{2} \Delta \varphi$, the length of a ray-segment of polarization $\pi$ between reflections. Furthermore, $T$ is the period of the periodic orbit. We finally obtain

$$
\operatorname{Tr} \mathbf{S}^{n} \approx \sqrt{\pi a \omega} \sum_{p o}^{(n)} A_{p o} e^{-i \omega T_{p o}+i n \pi / 2-i \pi / 4},
$$

where the sum is taken over all periodic rays with $n$ reflections and

$$
A_{p o}=\frac{\mathcal{A}_{p o}}{\sqrt{\frac{n_{p} C_{p}}{\cos \theta_{p}^{p o \sigma}}+\frac{n_{s} c_{s}}{\cos \theta_{s}^{p o}}}} .
$$

By taking the complex conjugate and the derivative with respect to $\omega$ of eq. (40), we finally obtain the spectral density to leading order in $1 / \omega$ as

$$
\begin{aligned}
d(\omega) & \approx \sqrt{\frac{a \omega}{\pi}} \sum_{n=1}^{\infty} \frac{1}{n} \sum_{p o}^{(n)} A_{p o} T_{p o} \cos \left(\omega T_{p o}-n \pi / 2+\pi / 4\right) \\
& =\sqrt{\frac{a \omega}{\pi}} \sum_{p p o} \frac{T_{p p o}}{\sqrt{\frac{n_{p} c_{p}}{\cos \theta_{p}^{p o}}+\frac{n_{s} c_{s}}{\cos \theta_{s}^{p O}}}} \sum_{r=1}^{\infty} \frac{\mathcal{A}_{p p o}^{r}}{r^{3 / 2}} \cos \left(r\left(\omega T_{p p o}-n_{p p o} \pi / 2\right)+\pi / 4\right) .
\end{aligned}
$$

The last expression is obtained after summing over orbits related by cyclic permutations of the symbol code $\boldsymbol{\pi}$ and the sum is now taken over all primitive periodic orbits (ppo) of arbitrary length, that is, over orbits not including repetitions and cyclic permutations. The second sum over $r$ then includes the repetitions.

We note in passing that the result (41) can also be derived from a generalization of the abelian trace formula [27, 28] valid for systems with continuous symmetries. Here the symmetry is used to integrate over families of orbits $\Gamma$. Due to the rotational symmetry in the disc, one obtains

$$
d_{\text {osc }}(\omega) \approx \sqrt{2 / \pi} \sum_{\text {orbit } \Gamma} \frac{\mathcal{A}_{\Gamma} T_{\Gamma}}{a_{\Gamma} \sqrt{|\partial \theta / \partial L|}} \cos \left(\omega T_{\Gamma}-\sigma_{\Gamma} \frac{\pi}{2}-\frac{\pi}{4}\right),
$$

where $T_{\Gamma}$ is the period of the orbit, $\sigma=\mu-1$ with $\mu$ the Maslov index, $a_{\Gamma}$ the order of the (possibly) discrete symmetry group of the orbit and finally $\mathcal{A}_{\Gamma}$ the product of the plane wave reflection coefficients for scattering at the boundary. Finally, $\partial \theta / \partial L$, (also called the anholonomy matrix), describing the negative change of perimeter angle due to a change of impact parameter is

$$
\frac{\partial \theta}{\partial L}=+2\left(\frac{n_{p}}{z_{p} \cos \theta_{p}}+\frac{n_{s}}{z_{s} \cos \theta_{s}}\right),
$$

in agreement with (38).

\subsection{Periodic orbit spectrum}

Eqn. (41) gives an explicit connection between periodic ray trajectories in the disc and the eigenfrequencies of the system. By taking a Fourier transform of $d(\omega)$ one should be able to recover the periodic ray solutions including orbits which change polarization along their path. To suppress high-frequency oscillations in the signal, we convolute Eq. (41) on 
both sides by a Gaussian test function as was also used in [⿶凵 . The smoothing depends on a parameter $\eta$ proportional to the width,

$$
w\left(z_{p}\right)=\frac{1}{\eta \sqrt{\pi}} e^{-\left(z_{p} / \eta\right)^{2}}, z_{p}=k_{p} a
$$

Figure 5 shows a comparison between a numerically calculated period spectrum obtained from the first 23000 eigenvalues of a disc using (14) and the approximative result (41), here for polyethylene with $\kappa=3.61$. The smooth part of the spectrum is removed and the density of eigenfrequencies is then Fourier transformed. This obtained period spectrum shows numerous peaks which fall roughly into three classes: orbits being of pure pressure, pure shear and mixed polarization type. In general the first class consists of the shortest orbits since the pressure waves have the fastest velocity. At $t \approx 3.2 \mathrm{msec}$, we have an infinite number of pressure orbits accumulating at the boundary; orbits of higher winding number like the pentagram at $t \approx 4.9 \mathrm{msec}$ can also be resolved. Next, orbits with segments of both pressure and shear polarization type arise. Again one finds accumulation towards a limit orbit with the pressure segments becoming tangential to the boundary around $t \approx 5.5 \mathrm{msec}$. We note here clear deviations of the actual numerical period spectrum from periodic orbit theory. Shear waves have incidence angles close to the critical angle and surface contributions become relevant.

We note, that for the first time, periodic orbits changing polarization along their paths could clearly be identified in a Fourier spectrum of an eigenspectrum of an elastic body. Quite characteristic is the decay of peak height as the orbits increase in length. Note, however, that the class of pure shear orbits with long periods show up as comparatively high peaks in the spectrum. This is due to the lack of mode-conversion when the shear segments turn towards tangential incidence with a reflection coefficient becoming a pure phase (23). Surface orbits like the pure Rayleigh orbit at $t \approx 12.2 \mathrm{msec}$ can also be identified.

\section{Summary and Discussion}

We have studied the in-plane eigenfrequency spectrum for the elastic wave equation in two spatial dimensions with circular boundaries. It was shown that the eigenmodes can be expressed in terms of periodic rays of an underlying billiard-like classical dynamics. The ray dynamics conserves angular momentum, the wave equation becomes separable in polar coordinates, accordingly. It was pointed out, however, that the existence of two wave modes with different velocities partially destroys the integrability of the problem; the ray dynamics for fixed angular momentum takes place on two different energy manifolds in phase space for the two polarization. The dynamics on each manifold is one-dimensional and thus integrable, transitions between the energy sheets at the boundary introduce a purely probabilistic component. The classical dynamics corresponding to the elastic wave equation is therefore not deterministic and thus not integrable in the sense of Hamiltonian dynamics.

By solving the wave equation explicitly and deriving high frequency approximations employing both the scattering matrix and the transfer operator, a connection between the wave dynamics in the disc for fixed angular momentum and the unitary propagation on a simple quantum graph could be established. Spectral correlations due to wave mixing manifest itself in a gap in the nearest neighbor spacing distribution and thus strong level 


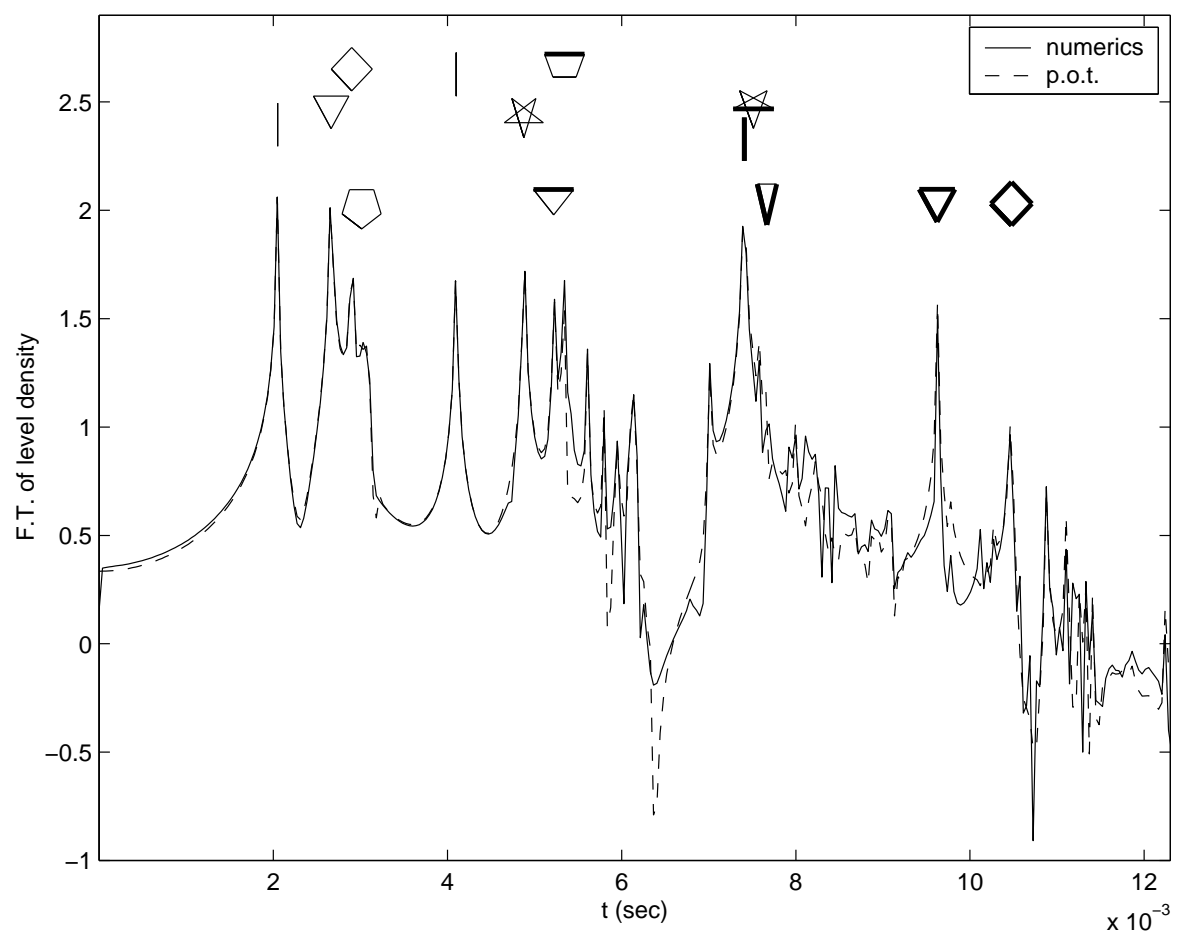

Figure 5: FFT of oscillating level density. The orbits are depicted with thin/fat lines for pressure/shear-polarization and are positioned according to their period. The periodic orbit theory ("p.o.t.") refers to (41). The smoothing parameter in (43) is chosen here as $\eta=0.2$. The actual material corresponds to polyethylene with $c_{p}=1950 \mathrm{~m} / \mathrm{s}$ and $c_{s}=540 \mathrm{~m} / \mathrm{s}$. Finally the disc radius is $a=1 \mathrm{~m}$. 
repulsion. Finally, the full level density was expressed in terms of periodic orbits which could be identified explicitly in the Fourier transform of the exact density of eigenstates.

The main corrections omitted in the high frequency approximations derived here occur for nearly tangential orbits both for pressure and shear components. This regime calls for a more refined approximation of the Bessel functions occurring in the traction matrix (15) as for example uniform approximations. Furthermore, periodic orbits accumulate at the boundary and the stationary phase approximation used to solve the integrals (35) breaks down. These corrections give rise to surface waves typical for free boundary conditions.

Furthermore, higher order terms in the reflection coefficients become important whenever the leading term $\alpha_{p s}$ vanishes, that is, for normal incident $\theta \rightarrow \pi / 2$ corresponding to $L \rightarrow 0$. Periodic orbits at $L=0$ having both $p$ and $s$ segments can indeed been identified in the Fourier spectrum fig. 5. A detailed analysis of these effects will be discussed elsewhere.

\section{Acknowledgment}

Financial support by the EPSRC and the EU - research training network Mechanics and Symmetry in Europe (MASIE) is gratefully acknowledged.

\section{A In-plane eigenfrequency spectrum for an elastic disc}

Due to the rotational symmetry of the disc, the wave equation separates in an angular and radial part. The eigenfunctions regular at the origin may be expressed in terms of Bessel functions; one writes the displacement field (2) as

$$
\mathbf{u}_{p}^{l}=\nabla\left(J_{l}\left(k_{p} r\right) e^{-i l \varphi}\right) \quad \mathbf{u}_{s}^{l}=\nabla \times \hat{\mathbf{z}}\left(J_{l}\left(k_{s} r\right) e^{-i l \varphi}\right)
$$

and $p, s$ refers to pressure and shear polarization as usual, (also called "primary" and "secondary" wave in seismology referring to the time of arrival of these waves). A general interior eigenfunction can be expanded in these displacement fields, that is,

$$
\mathbf{u}_{l}=a_{1} \mathbf{u}_{p}^{l}+a_{2} \mathbf{u}_{s}^{l} .
$$

To fulfill the free boundary condition, the traction of $\mathbf{u}_{l}$ has to vanish at the boundary, that is,

$$
\mathbf{t}\left(\mathbf{u}_{l}\right)=a_{1} \mathbf{t}\left(\mathbf{u}_{p}^{l}\right)+a_{2} \mathbf{t}\left(\mathbf{u}_{s}^{l}\right)=0 .
$$

Writing the traction in terms of its radial and angular direction, one obtains

$$
\begin{aligned}
0 & =a_{1} \mathbf{t}_{r}\left(\mathbf{u}_{p}^{l}\right)+a_{2} \mathbf{t}_{r}\left(\mathbf{u}_{s}^{l}\right) \\
0 & =a_{1} \mathbf{t}_{\varphi}\left(\mathbf{u}_{p}^{l}\right)+a_{2} \mathbf{t}_{\varphi}\left(\mathbf{u}_{s}^{l}\right) .
\end{aligned}
$$

Expressed as a matrix equation this becomes

$$
\left(a_{1} a_{2}\right) \cdot \mathbf{t}_{l}=\mathbf{0}
$$

where we have collected the coordinates of both polarizations in a matrix

$$
\begin{aligned}
\mathbf{t}_{\mathbf{l}} & =\left[t_{\pi i}^{l}\right] \\
& =\left(\begin{array}{cc}
\left(l^{2}-\frac{1}{2} z_{s}^{2}\right) J_{l}\left(z_{p}\right)-z_{p} J_{l}^{\prime}\left(z_{p}\right) & i l\left(J_{l}\left(z_{p}\right)-z_{p} J_{l}^{\prime}\left(z_{p}\right)\right) \\
i l\left(J_{l}\left(z_{s}\right)-z_{s} J_{l}^{\prime}\left(z_{s}\right)\right) & z_{s} J_{l}^{\prime}\left(z_{s}\right)-\left(l^{2}-\frac{1}{2} z_{s}^{2}\right) J_{l}\left(z_{s}\right)
\end{array}\right)
\end{aligned}
$$


with $i=r$ or $\varphi$ and $\pi=p$ or $s$ and thus $t_{\pi i}=\mathbf{t}_{i}\left(\mathbf{u}_{\pi}\right)$. We set as usual $z_{\pi}=a k_{\pi}$ with $a$, the radius of the disc. A superposition of these two polarizations fulfills the boundary condition only when

$$
\operatorname{det}\left(\mathbf{t}_{l}\right)=0
$$

which is the eigenfrequency condition (14). For more details see for example [29] in which an expression equivalent to (48) is derived.

\section{B S-matrix}

\section{B.1 S-matrix in terms of traction operators}

Assume a general boundary condition

$$
\boldsymbol{\Omega}(\mathbf{u})=\mathbf{0}
$$

with $\mathbf{u}$ an $n$-dimensional vector wave function and $\boldsymbol{\Omega}$ a linear operator. Denote $\mathbf{u}_{i}$ the wave field being non-zero in its $i$-th component only with $i \in\{1, \ldots, n\}$. The scattering process of an incoming pure $i$-wave, $\mathbf{u}_{i}^{-}$, may then be described as

$$
\mathbf{u}=\mathbf{u}_{i}^{-}+\sum_{j} S_{i j} \mathbf{u}_{j}^{+}
$$

where $\mathbf{u}_{j}^{+}$denotes outgoing pure polarizations. The Eq. (51) must satisfy the boundary condition (50) for all $i$. Solving for the scattering matrix, one obtains

$$
\mathbf{S}=-\boldsymbol{\Omega}\left(\mathbf{u}^{-}\right) \cdot\left(\boldsymbol{\Omega}\left(\mathbf{u}^{+}\right)\right)^{-1}
$$

The operator $\boldsymbol{\Omega}(\mathbf{u})$ may be represented in matrix form

$$
\left(\Omega\left(u_{i}\right)\right)_{j}
$$

where the index $i$ represents the component of the vector field $\mathbf{u}$ and $j$ denotes the vector component of the operator $\boldsymbol{\Omega}$, (that is, $\hat{x}, \hat{y}$ or $\hat{r}, \hat{\varphi}$ in two dimensions).

In our case $\boldsymbol{\Omega}(\mathbf{u})$ is given by the traction $\mathbf{t}(\mathbf{u})$ (ब) in polar coordinates. The analytic form is obtained from (48) using $H_{l}^{(1)}, H_{l}^{(2)}$ instead of $J_{l}$.

\section{B.2 The high frequency limit $\omega \gg 1$ for $b_{p}=l / z_{p}<1$}

Starting from the Eq. (16) expressing the scattering matrix $\mathbf{S}_{l}$ in terms of traction matrices, we will employ the oscillatory Debye approximation for the Hankel functions entering $\mathbf{t}^{ \pm}$, that is,

$$
H_{l}^{(1)}(z) \sim \sqrt{\frac{2}{\pi Q}} e^{i \phi} ; \quad H_{l}^{(2)}(z) \sim \sqrt{\frac{2}{\pi Q}} e^{-i \phi} ; \quad H_{l}^{(1)^{\prime}}(z) \sim i \frac{Q}{z} H_{l}^{(1)}(z)
$$

with

$$
Q=\sqrt{z^{2}-l^{2}} \text { and } \phi=Q-\pi / 4-l \arccos \frac{l}{z} .
$$

Here, $z$ is either $z_{p}$ or $z_{s}$ with $z_{\pi}=a k_{\pi}$ as usual. The traction matrices $\mathbf{t}^{ \pm}$are obtained from (15) by replacing the Bessel functions in terms of outgoing and incoming Hankel 
functions, respectively. Inserting the Debye approximation and separating $\mathbf{t}$ in amplitude and phase, we obtain [30]

$$
\mathbf{t}^{ \pm}=\left(\begin{array}{cc}
e^{ \pm i \phi_{p}} & 0 \\
0 & e^{ \pm i \phi_{s}}
\end{array}\right) \cdot \mathbf{G}^{ \pm} \cdot \mathbf{Z}^{ \pm}
$$

Here

$$
\mathbf{G}^{ \pm}=\left(\begin{array}{cc}
\sqrt{\frac{2}{\pi Q_{p}}} & 0 \\
0 & \sqrt{\frac{2}{\pi Q_{s}}}
\end{array}\right)
$$

and

$$
\mathbf{Z}^{+}=\left(\begin{array}{cc}
\left(l^{2}-z_{s}^{2} / 2\right)-i Q_{p} & i l\left(1-i Q_{p}\right) \\
i l\left(1-i Q_{s}\right) & i Q_{s}-\left(l^{2}-z_{s}^{2} / 2\right)
\end{array}\right)
$$

whereas $\mathbf{Z}^{-}$has the same form as $\mathbf{Z}^{+}$apart from replacing $Q_{\pi}$ by $-Q_{\pi}$. Hence,

$$
\mathbf{S}=\left(\begin{array}{cc}
e^{-i \phi_{p}} & 0 \\
0 & e^{-i \phi_{s}}
\end{array}\right) \cdot \boldsymbol{\alpha} \cdot\left(\begin{array}{cc}
e^{-i \phi_{p}} & 0 \\
0 & e^{-i \phi_{s}}
\end{array}\right)
$$

with the unitary matrix $\boldsymbol{\alpha}$ defined as

$$
\boldsymbol{\alpha}=-\mathbf{G}^{-} \cdot \mathbf{Z}^{-} /\left(\mathbf{G}^{+} \cdot \mathbf{Z}^{+}\right) .
$$

A straightforward but tedious expansion of $\boldsymbol{\alpha}$ in terms of the wave numbers $k_{\pi}$, expressing $l$ and $Q_{\pi}$ in terms of the incidence angle $\theta_{\pi}$, that is, $l=-z_{\pi} \sin \theta_{\pi}$ and $Q_{\pi}=z_{\pi} \cos \theta_{\pi}$, one obtains indeed the reflection coefficients (6) in leading order. The limit taken corresponds to letting $k_{\pi} \rightarrow \infty$ and $|l| \rightarrow \infty$ but keeping their ratio fixed. As discussed above this ratio corresponds to fixed impact parameter/incidence angle. This finally reproduces formula (19). All the formulas are given here for negative angular momentum; choosing $l=z_{\pi} \sin \theta_{\pi}$ positive changes the sign of the off-diagonal components in (15). This does not alter the eigenfrequency condition (14) reflecting the degeneracy of the spectrum with respect to the sign change in $l$.

\section{References}

[1] Y. Safarov and D. Vassiliev, in Spectral Theory of Operators, edited by S. Gindikin, AMS Translations, ser.2, 150, p. 55 (1992).

[2] M. Dupuis, R. Mazo, and L. Onsager, Journ. of Chem. Phys. 33, 1452 (1960).

[3] M.C. Gutzwiller, Chaos in Classical and Quantum Mechanics (Springer, New York 1990).

[4] M. Brack and R.K. Bhaduri, Semiclassical Physics (Addison-Wesley, Reading 1997).

[5] M.V. Berry and M. Tabor J. Phys. A 10, 371 (1977).

[6] M.C. Gutzwiller, J. Math. Phys. 12, 343 (1971).

[7] L.D. Landau and E.M. Lifshitz, Theory of Elasticity (Pergamon, Oxford 1959).

[8] D. Biswas, Phys. Rev. E 54, 1232 (1996). 
[9] L. Couchman, E. Ott and T.M. Antonsen, Jr., Phys. Rev. A 46, 6193 (1992).

[10] A. Wirzba, N. Sondergaard, and P. Cvitanović, nlin.CD/0108053, submitted to Phys. Rev. Lett. .

[11] M.L. Mehta, Random Matrices (2nd ed., Academic Press, New York 1991).

[12] T. Guhr, A. Müller-Groeling, and H.A. Weidenmüller, Physics Report 299, 189 (1998).

[13] O. Legrand, C. Schmitt, and D. Sornette, Europhys. Lett.,18, 101 (1992).

[14] C. Ellegaard, T. Guhr, K. Lindemann, J. Nygård, and M. Oxborrow, Phys. Rev. Lett. 77, 4918 (1996).

[15] K. Schaadt, G. Simon, and C. Ellegaard, Physica Scripta, 90, 231 (2001).

[16] T. Kottos and U. Smilansky, Phys. Rev. Lett. 79, 4794 (1997).

[17] G. Tanner, J. Phys. A 33, 3567 (2000).

[18] A. Bedford and D.S. Drumheller, Introduction to elastic wave propagation, (John Wiley and Sons, Chichester, 1994).

[19] Y.H. Pao and C.C. Mow, Diffraction of Elastic Waves and Dynamic Stress Concentrations (Rand Corporation, New York 1971).

[20] J.D. Achenbach, A.K. Gautesen and H. McMaken, Ray Methods for Waves in Elastic Solids (Pitman, Boston 1982).

[21] M. Bonnet, Boundary Integral Equation Methods for Solids and Fluids, (John Wiley and Sons, Chichester 1995).

[22] E.B. Bogomolny, Nonlinearity 5, 805 (1992).

[23] U. Smilansky, in Les-Houches Summer School on mesoscopic quantum physics, edited by E. Akkermans et al., (North-Holland 1994), p. 373.

[24] F. Barra and P. Gaspard, J. Stat. Phys. 101, 283 (2000).

[25] M.V. Berry and C.J. Howls, Proc.Roy.Soc.Lond.A 447, 527, (1994).

[26] U. Smilansky and I. Ussishkin, J. Phys. A: Math. Gen. 29, 2587 (1996).

[27] S. Creagh and J.D. Littlejohn, J. Phys. A: Math. Gen. 251643 (1992).

[28] S. Creagh, Ann. of Physics 248, 60 (1996).

[29] M. Kitahara, Boundary Integral Equation Methods in Eigenvalue Problems of Elastodynamics and Thin Plates, (Elsevier, Amsterdam 1985).

[30] A. Wirzba, private communication. 